\title{
RAMPEX - a new spin experiment
}

\author{
Yu. Arestov \\ Institute for High Energy Physics, 142284 Protvino, Moscow region, Russia; \\ E-mail: arestov@mx.ihep.su \\ (presented for HELION97, 20-24 Jan 1997, Kobe, Japan)
}

\begin{abstract}
RAMPEX - Russian-AMerican Polarization EXperiment- is dedicated to studies of one-spin asymmetries which have twist-3 and also twist- 2 origin, in hard and semihard inclusive processes on the polarized propane-diol target. A special consideration has been given for the prospects of using polarized ${ }^{3} \mathrm{He}$ target. The studies will be performed at the Serpukhov accelerator at $70 \mathrm{GeV} / \mathrm{c}\left(p\right.$ beam) and $40 \mathrm{GeV} / \mathrm{c}\left(\pi^{-}\right.$ beam).
\end{abstract}

Key words: polarized target, magnetic spectrometer, calorimetry, DAQ, spin experiment, polarization asymmetry

PACS: 01.52.+r, 07.05.Hd, 07.20.Fw, 07.77.Ka

\section{Introduction.}

RAMPEX - Russian-AMerican Polarization EXperiment- presents a program of studies of one-spin effects in hadron processes. A significant part of the investigations will be performed for the first time. This program includes $p p_{\uparrow}$ interactions at $70 \mathrm{GeV} / \mathrm{c}$, and as an option $\pi^{-} p_{\uparrow}$ interactions at $40 \mathrm{GeV} / \mathrm{c}$, at the Serpukhov accelerator. Conceptually RAMPEX will try to form a new approach in interpreting experimental results on one-spin asymmetries in hadron processes.

Quantum chromodynamics as a model of strong interactions is commonly used in interpretation of experimental data. The most successful descriptions hold for those effects which correspond to the leading twist-2 contributions. These include collider data on hard photon and jet production at large $p_{T}$, on lepton pair production with large $M$ etc.

The higher-twist contributions are less familiar to most of physicists. The 
twist-3 phenomena relate to the one-spin processes, such as hard and semihard hadron production on polarized protons in reactions

$$
p p_{\uparrow} \rightarrow h+X
$$

at high initial energies. Though one-spin asymmetries were studied experimentally at various energies (see for example review [1]), no special attention was paid to the twist-3 origin of the one-spin asymmetries.

There are also subtle twist-2 effects in the double inclusive processes

$$
p p_{\uparrow} \rightarrow h_{1}+h_{2}+X
$$

which have not yet been studied.

The spin theoretical community is highly interested in the new results concerning these twist-3 and twist-2 hadron processes $(1,2)$ (see review [2]). The forthcoming RAMPEX stimulates them to formulate new concepts for one-spin asymmetries.

Analysis of the whole set of experimental measurements which are available between beam momenta of 6 and $200 \mathrm{GeV} / \mathrm{c}$ (for refs. see [1]), results in the following conclusions:

- at any initial energy under study, the one-spin asymmetry can reach sizeable values;

- a serious enigma is the zero values of $A_{N}\left(p_{T}\right)$ in a very wide $p_{T}$-interval in E704 experiment at $200 \mathrm{GeV} / \mathrm{c}$;

- the experimental measurements are rather mosaic, and mostly they cannot be compared;

- a new experiment is highly desirable in which a complex physical program can be performed on $A_{N}\left(x_{F}, p_{T}\right)$ for different particles and in different kinematical regions.

\section{Physical motivation of RAMPEX.}

Normally the experimental community is familiar with the polarized parton densities $g_{1}(x)$ and $g_{2}(x)$ probed in DIS. However the intrinsic nucleon structure is described also by other functions, of twist-2 and twist-3 in particular (see for example [3]).

In RAMPEX we shall measure the twist-3 asymmetries in single hadron production in (1) and we shall try to discover the more subtle twist-2 correlations 
in two-particle production processes in (2). In both cases $h$ 's denote $\pi, K, \ldots$ As is commonly believed, the one-spin asymmetries in hard and semi-hard hadron production processes can be used, in appropriate phenomenology, to obtain information on new spin-dependent quark distributions $h_{L}(x)$ and $h_{1}(x)$, of twist-3 and twist-2 respectively. They are both chiral-odd distribution functions.

The relevant theoretical problems were discussed at the RAMPEX Round Table at SPIN96 [2].

The decaying $\Lambda$-hyperons are good self-analysing polarization tools. The measurment of the final-state $\Lambda$ polarization in the reaction

$$
p+p_{\uparrow} \rightarrow \Lambda_{\uparrow}+X
$$

will allow to study the proton $-\Lambda$ spin correlations. We shall also perform first polarization asymmetry measurements in the production processes of the resonanses $K_{890}^{*}$ and $\phi$ containing $s$ quarks.

\section{General layout.}

The full version of the experimental setup includes two arms (fig. 1). One arm consists of the magnet spectrometer, two Čerenkov counters Č1, Č2 to identify charged particles, an electromagnetic calorimeter EC1 and a hadron calorimeter $\mathrm{HC}$. The magnet spectrometer consists of the magnet $\mathrm{M}$ and five proportional chambers PC1-PC5. In fig. 1 this arm makes an angle of $9^{\circ}$ with the beam line corresponding to $90^{\circ}$ in $\mathrm{cms}$. This arm will be also rotated to a smaller angle close to $0^{\circ}$ to detect particles with large $x_{F}$ and to a larger angle to detect particles with negative $x_{F}$. Numerical estimations of acceptances and efficiences show that the angle values near 80 and $300 \mathrm{mrad}$ are optimal for these measurements. The second arm of the setup consists only of the finegranulated electromagnetic calorimeter EC2 which is placed symmetrically to the beam line and makes angle $-9^{\circ}$ or smaller. 


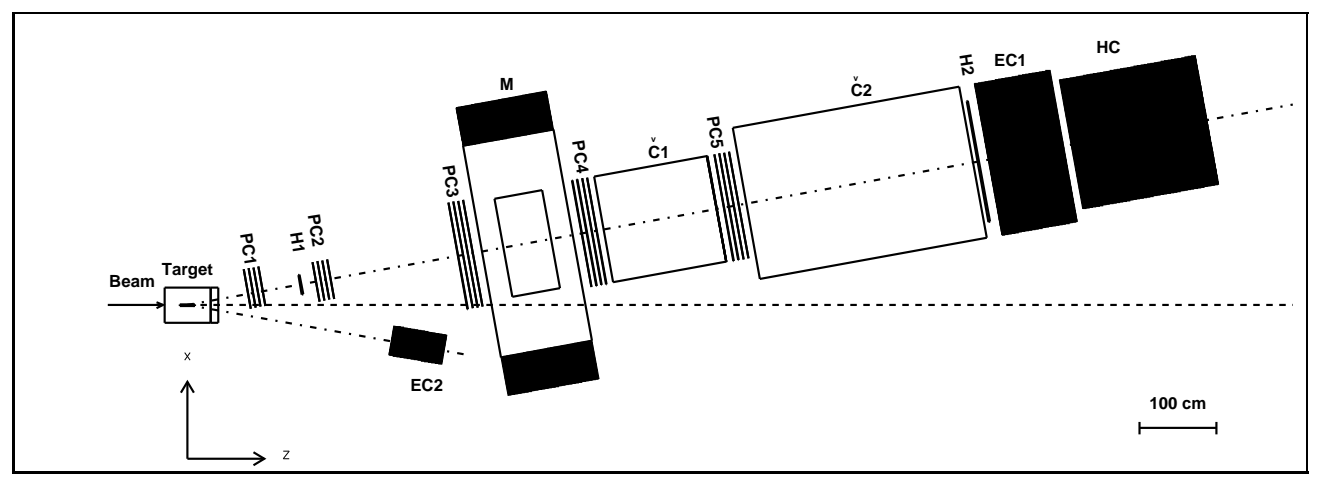

Fig. 1. Layout of experimental setup RAMPEX: PC1-PC5 - blocks of proportional chambers, M - analysing magnet, H1, H2 - trigger hodoscopes, Č1, Č2 - threshold Čerenkov counters, EC1, EC2 - electromagnetic calorimeters, HC - hadron calorimeter.

3.0.0.1 Beam. The $70 \mathrm{GeV} / \mathrm{c}$ unpolarized proton beam is extracted from the accelerator with a bent $\mathrm{Si}$ crystal [4], and the measurements with this beam will take a major part of the experimental program. The $40 \mathrm{GeV} / \mathrm{c} \pi^{-}$ extracted beam will be also used. The pion/proton beam intensity is $5 \cdot 10^{6}$ in a 1-second spill with a 9 -second interval between spills.

3.0.0.2 Polarized target. Propane-diol $\mathrm{C}_{3} \mathrm{H}_{8} \mathrm{O}_{2}$ fills a cavity $20 \mathrm{~mm}$ in diameter and $200 \mathrm{~mm}$ in length. The polarization of the hydrogen nuclei is about $80 \%$ on average. The dilution factor defined as a ratio of the number of the target nuclei to the number of polarized nuclei depends on a type of detected particle and kinematics, and varies between about 6 to 10 . The target contains $9.3 \cdot 10^{24}$ nucleons $/ \mathrm{cm}^{2}$. The luminosity of the experiment is estimated as $\mathcal{L} \sim 5 \cdot 10^{31} \mathrm{~cm}^{-2}$ spill $^{-1}$.

3.0.0.3 Magnetic spectrometer. The magnetic spectrometer includes the analysing magnet $\mathrm{M}$ and 5 blocks of multiwire proportional chambers $\mathrm{PC} 1$ - PC5. Each block contains four coordinate planes: orthogonal $x$ and $y$ planes and also $u$ and $v$ planes which are inclined to $\pm 10^{\circ}$ with respect to $y$ axis. The magnet $M$ has the aperture of $1.3 \times 0.62 \mathrm{~m}^{2}$ and a length of $0.63 \mathrm{~m}$. The integral of the magnetic field is $1.0 \mathrm{Tm}$. The magnet center is placed $4.5 \mathrm{~m}$ from the target center.

Two blocks of proportional chambers PC1 and PC2 with the transverse size $530 \times 384 \mathrm{~mm}^{2}$ are located at distances 0.9 and $2 \mathrm{~m}$ from the target center, respectively. Three blocks of chambers PC3 - PC5 of size $1422 \times 898^{2}$ are placed at the distances 3.7, 5.2 and $7.2 \mathrm{~m}$ from the target.

The tracking system will allow us to measure charged particle momenta with 
an accuracy $\Delta p / p=1.7 \cdot 10^{-3} p+2 \cdot 10^{-3}$ and to reconstruct the straight-line track $x=x_{0}+a_{x} z$ with an accuracy $\delta x_{0}=2, \delta a_{x}=8.8 \cdot 10^{-4}$.

3.0.0.4 Charged particle identification. Particle types for $\pi, K, p$ and $\bar{p}$ are determined with the help of two threshold multi-channel Cerenkov counters $\check{C}_{1}$ and $\check{C ̌}_{2}$ [5]. The counter Č1 has 8 channels $(4 \times 2)$ and it is filled with freon-12 at $1 \mathrm{~atm}$. The 16 -channel counter Č2 $(8 \times 2)$ is filled with nitrogen also at $1 \mathrm{~atm}$. Combinations of two counters can identify $\pi^{ \pm}$with momenta $3.1 \div 20 \mathrm{GeV} / \mathrm{c}$, and $K^{ \pm}$and $p^{ \pm}$from 10 to $20 \mathrm{GeV} / \mathrm{c}$. The aperture and the length of $\check{\mathrm{C}} 1$ (Č 2$)$ are $1.2 \times 0.9 \mathrm{~m}^{2}$ and $1.5 \mathrm{~m}\left(1.6 \times 0.88 \mathrm{~m}^{2}\right.$ and $\left.3.0 \mathrm{~m}\right)$.

3.0.0.5 Electromagnetic (EM) calorimetry. Two EM calorimeters (EC1 and EC2 in fig. 1) will detect EM showers. The EC1 cells are made of $\mathrm{Pb}$ and scintillator and have sizes $38 \times 38 \mathrm{~mm}^{2}$ (center) and $76 \times 76 \mathrm{~mm}^{2}$ (periphery). The EC2 cells of size $38 \times 38 \mathrm{~mm}^{2}$ are made of $\mathrm{PbWO}_{4}$ [6]. The energy resolutions for EC1 and EC2 are $\sigma_{E} / E \approx 9 \% / \sqrt{E}+0.5 \%$ and $\sigma_{E} / E \approx 3 \% / \sqrt{E}+0.5 \%$ respectively.

3.0.0.6 Hadron calorimeter. The hadron calorimeter HC of compensating type [7] will be used to detect $K_{L}^{0}$ and neutrons and also as an element of the trigger system. The $10 \mathrm{~cm} \times 10 \mathrm{~cm} \mathrm{Pb+Sci} \mathrm{sandwiches} \mathrm{form} \mathrm{a}$ matrix of $18 \times 12$ modules (216 channels total) resulting in energy resolution $\sigma_{E} / E \approx 57 \% / \sqrt{E}$. The ratio of electron to hadron signals is equal to $e / h=1.01 \pm 0.03$.

3.0.0.7 DAQ and trigger. Data acquisition system and trigger electronics are being worked out to fit the data flow of $\sim 2000$ events per burst with the event size $\sim$ of $1 \mathrm{Mb}$. The zero level trigger is arranged to strobe information from the trigger hodoscopes. The simplest first level trigger for the charged arm of the spectrometer is a signal coincidence from the two trigger hodoscopes. A special work is made for triggering with hadron calorimeter using the proportionality of the signal $E_{x}=\sum E_{i} \cdot \sin \theta_{x_{i}}$ (summation over counters) to the transverse momentum $p_{T}$. The overall one-charged particle trigger has been worked out and tested. The multi-particle trigger is in progeress. The details of triggering can be found in [8].

3.0.0.8 First accelerator runs. The first test run was performed in Fall'96. The aim was to test electromagnetic calorimeter and the related systems of the programming shell. 320 of $1200 \mathrm{EC} 1$ modules made of $\mathrm{Pb}+$ scintillator sandwiches were examined and showed the overall energy resolution of $\sigma=9 \% / \sqrt{E}$. 
The EC1 related data acquisition was carefully tested including such elements as read-out electronics, data flow, calibration, LED based monitoring system and HV power supply. While being tested EC1 moved in two dimensions in the vertical plane with high accuracy controlled by a special program. The off-line analysis was also undertaken. The results of that testing run are regarded as quite satisfactory.

The second test run has been scheduled for March'97. The program includes looking over the remaining $880 \mathrm{EC} 1$ modules and first tests of the tracking system. A certain work will be made to improve parameters of the $70-\mathrm{GeV} / \mathrm{c}$ proton beam extracted with the bent crystal in the 14th channel.

The first data-taking is being planned for the end of the Fall'97 run supposing detection of $\pi^{o}$ and $\eta$ signals. A major part of this run will be dedicated to the further tests of the tracking system and to the first launch of the whole setup.

\section{$4{ }^{3}$ He target prospects at RAMPEX.}

Within any reasonable accelerator run duration, the physical capabilities of RAMPEX is generally restricted by the properties of the polarized propane-diol target. It becomes opaque at the beam fluxes more than $10^{7}$. So the luminosity $k \cdot 10^{31} \mathrm{~cm}^{-2} \mathrm{spill}^{-1}$ is considered as a maximum. Besides the luminosity related to the collisions with the polarized protons in the target is less by a factor of 10 .

Using the internal polarized ${ }^{3} \mathrm{He}$ target in the $70-\mathrm{GeV} / \mathrm{c}$ proton ring would provide new possibilities in the twist-3 and twist- 2 studies at RAMPEX. In particular, the presence of polarized neutrons will be very instructive for search of the flavor dependence of the asymmetry.

A comparison of polarization effects in inclusive production of particles with various quark content is of special interest. With the internal ${ }^{3} \mathrm{He}$ target we could compare one-spin asymmetries on polarized protons and polarized neutrons with appropriate statistics in the following reactions:

$$
\begin{aligned}
p+p_{\uparrow}\left(n_{\uparrow}\right) & \rightarrow \pi^{0}+X(d \bar{d}) \\
& \rightarrow K_{s}^{0}+X(d \bar{s}+\bar{d} s) \\
p+p_{\uparrow}\left(n_{\uparrow}\right) & \rightarrow \pi^{+}+X(u \bar{d}) \\
& \rightarrow K^{+}+X(u \bar{s}) \\
p+p_{\uparrow}\left(n_{\uparrow}\right) & \rightarrow \pi^{-}+X(d \bar{u}) \\
& \rightarrow K^{-}+X(s \bar{u}),
\end{aligned}
$$


The most promising are the kinematic regions at $x_{F}=0$ and/or at the negative $x_{F}$. The one-spin asymmetry on the subprocess level which originates from perturbative QCD is proportional to the mass of the polarized quark. The masses of $u, d$ quarks are negligibly small, and it is commonly believed that the strange quarks in polarized proton are weakly polarized.

On the contrary, the one-spin asymmetry originating from the twist-3 contributions is proportional to the mass parameter $\mu_{\text {hadr }}$ due to the long-distance interactions [9]. As the fragmentation properties may differ for pions and kaons, the effective size of this region, $\sim 1 / \mu_{\mathrm{hadr}}$, may also vary, thus resulting in a flavor dependence of the one-spin asymmetries.

With the ${ }^{3}$ He target the higher statistics can be achieved at the large- $p_{T}$ twist3 studies in the processes (1) and the very subtle twist-2 asymmetries in the processes (2). The proton beam intensity in the ring is equal to $I_{\text {beam }}=10^{13}$ protons per spill. The length of the spill is 9 seconds with the 2 -second flat part of the maximum accelerating field in the middle of the spill. The frequency of the beam revolutions during this 2-second window is equal to $n=4 \cdot 10^{5}$ spill ${ }^{-1}$. Assuming the ${ }^{3} \mathrm{He}$ target parameters to be the same as was reported by the RIKEN group at SPIN96 in Amsterdam [10], that is the density of $5 \cdot 10^{14}$ atoms $/ \mathrm{cm}^{3}$ and length of $10 \mathrm{~cm}$, one obtains the target density of $I_{T}$ $=1.5 \cdot 10^{16}$ nucleons $/ \mathrm{cm}^{2}$.

This results in luminosity $\mathcal{L}=n I_{\text {beam }} I_{T}=6 \cdot 10^{34} \mathrm{~cm}^{-2} \mathrm{~s}^{-1}$ which is higher than the current RAMPEX luminosity by a factor of $10^{3}$. In a standard one-month run $\left(2.8 \cdot 10^{5}\right.$ spills $)$ this corresponds to the statistics of $10^{4}$ events per picobarn. At this level of statistics the twist-3 asymmetry measurements in (1) seem to be reliable up to $p_{T}=4 \mathrm{GeV} / \mathrm{c}$.

\section{References}

[1] S.B. Nurushev, Talk given at Int. Workshop on High Energy Spin Physics, Sept. 1995, Protvino (Proc. published in 1996, Ed. by S.B. Nurushev).

[2] Yu. Arestov, Review of the RAMPEX Round Table, In Proc. Int. Symp. on High Energy Spin Physics, Amsterdam, Sept. 1996.

[3] R.L. Jaffe and X.Ji, Phys.Rev.Lett. 67 (1991) 552; X.-D. Ji, Phys.Lett. B284 (1992) 137.

[4] A. A. Aseev et a.l Nucl. Instr. Meth. A330 (1993) 39.

[5] A.M.Aleev et al., Preprint JINR P13-94-520, Dubna, 1994.

[6] O.V.Buyanov et al., Nucl. Instr. Meth. A 349 (1994) 62-69.

[7] G.Alekseev et al., Preprint IHEP 92-36, 1992. 
[8] RAMPEX, Proposal of Experiment, Preprint IHEP (in press).

[9] A.V.Efremov and O.V.Teryaev, Sov. J. Nucl. Phys. 39(1984)962; Yad. Fiz. 39(1984)1517; Phys Lett 150B(1985)383.

[10] T Uesaka et al., Development of Polarized ${ }^{3}$ He Gas Target at RIKEN, SPIN96 (12th Int. Symp. on High Energy Spin Physics, Sept. 1996, Amsterdam; Book of Abstracts, p.287. 